\title{
MOMENT PREFERENCES AND POLYNOMIAL UTILITY *
}

\author{
Sigrid M. MÜLLER \\ University of Mannheim, 6800 Mannheim 1, Federal Republic of Germany
}

Mark J. MACHINA

University of California, San Diego, La Jolla, CA 92093, USA

Received 12 January 1987

Accepted 9 February 1987

This paper presents a direct algebraic (i.e., non-calculus) proof of the well-known equivalence of $m$-moment preferences and $m$-degree polynomial utility for an expected utility maximizer.

\section{Introduction}

Everyone knows that an expected utility maximizer will evaluate probability distributions on the basis of their first two moments ${ }^{1}$ if and only if his or her von Neumann-Morgenstern utility function $U(\cdot)$ is quadratic, and similarly for higher numbers of moments and higher degree polynomials. ${ }^{2}$ Although the implication

$\{U(\cdot)$ is an $m$-degree polynomial $\} \rightarrow$ only the first $m$ moments matter $\}$

is obvious and its converse

$\{$ only the first $m$ moments matter $\} \rightarrow\{U(\cdot)$ is an $m$-degree polynomial $\}$

can be established by a Taylor series argument if $U(\cdot)$ is assumed to be differentiable of a sufficiently high order, we have been puzzled by the absence (to our knowledge) of a direct non-calculus based proof of this latter result in the literature. The purpose of this letter is to provide such a proof, which we hope will help reveal the simple algebraic nature of this result. The next section presents the basic idea in the mean-variance case, and section 3 offers a formal proof for the case of $m$ moments.

* We are grateful to Joel Sobel and Michael Rothschild for helpful discussions on this material.

1 Although our main result (section 3) will be presented in terms of the mean and higher absolute moments $\mathrm{E}[X], \mathrm{E}\left[X^{2}\right]$, $\mathrm{E}\left[X^{3}\right], \ldots$, it could be alternatively expressed in terms of the mean and higher central moments $\mathrm{E}[X], \mathrm{E}\left[(X-\mathrm{E}[X])^{2}\right]$, $\mathrm{E}\left[(X-\mathrm{E}[X])^{3}\right], \ldots$, as in the mean variance case of section 2 .

2 Discussions of moment preferences in economics and finance include Bierwag (1974), Borch (1969, 1973, 1974), Hanson and Menezes (1971), Levy (1974), Machina and Rothschild (1987), Samuelson (1970), Scolt and Horvath (1980), Tobin $(1958,1969)$, Tsiang $(1972,1974)$ and the references cited in there. Although it will have no bearing on our results, it is important to recall that the assumption of polynomial utility typically requires a restriction on the set of allowable outcomes if utility is to be non-decreasing. 


\section{Mean-variance preferences and quadratic utility}

It is clear that a continuous function $U(\cdot)$ will possess a constant first difference, i.e., will satisfy

$[U(x+\delta)-U(x)] \underset{x, \delta}{\equiv}[U(\delta)-U(0)]$

if and only if it is linear (i.e., affine). Similarly, a continuous $U(\cdot)$ will possess a constant second difference, i.e., will satisfy

$[U(x+2 \delta)-U(x+\delta)]-[U(x+\delta)-U(x)] \underset{x, \delta}{\equiv}[U(2 \delta)-U(\delta)]-[U(\delta)-U(0)]$

if and only if it is quadratic.

Consider the two probability distributions illustrated in fig. 1, where $x$ and $\delta$ are arbitrary. Since each has a mean of $x / 2+\delta$ and a variance of $x^{2} / 4+\delta^{2} / 2$, the assumption of mean-variance preferences implies that they will yield equal expected utility, so that we have

$\frac{1}{4} U(0)+\frac{1}{4} U(2 \delta)+\frac{1}{2} U(x+\delta) \underset{x, \delta}{\equiv} \frac{1}{2} U(\delta)+\frac{1}{4} U(x)+\frac{1}{4} U(x+2 \delta)$.

But since (5) is equivalent to (4), it follows that $U(\cdot)$ must be quadratic.

\section{3. $m$-moment preferences and $\boldsymbol{m}$-degree polynomial utility}

The two distributions in fig. 1 were chosen so that (i) they possessed the same first two moments for all $x$ and $\delta$, and (ii) equating their expected utility for all $x$ and $\delta$ implied that $U(\cdot)$ possessed a constant second difference and hence was quadratic. Not surprisingly, this approach can be extended to the general case of $m$ moments. Specifically, we know from Aczèl $(1966$, p. 130) that a continuous
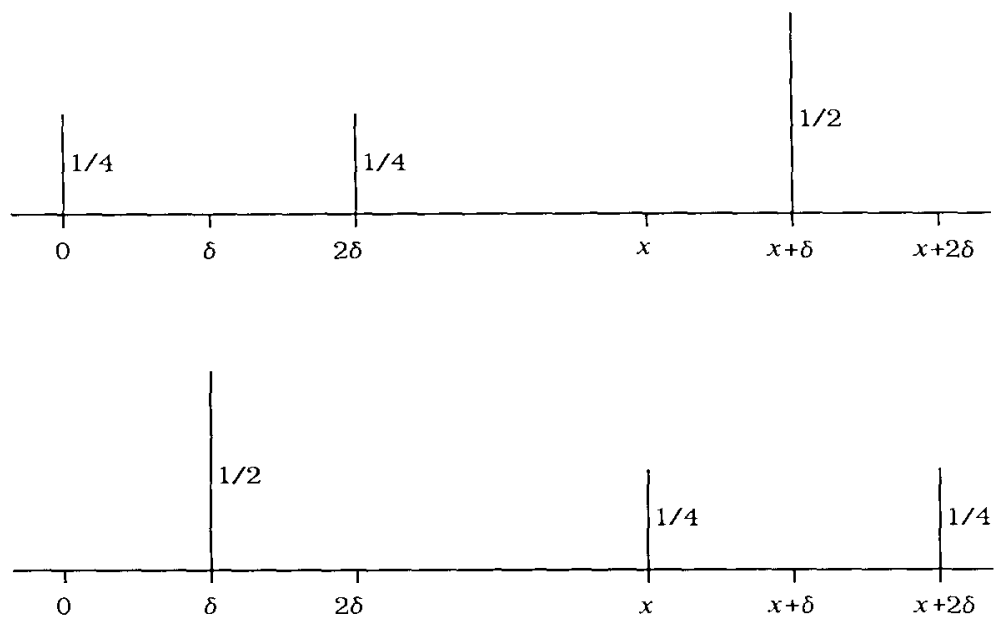

Fig. 1. Probability distributions for the mean-variance case. 
function $U(\cdot)$ will be a polynomial of at most degree $m$ if and only if it possesses a constant $m$ th difference, i.e., if and only if

$$
\sum_{k=0}^{m}(-1)^{k} \cdot\left(\begin{array}{c}
m \\
k
\end{array}\right) \cdot U(x+k \delta) \equiv \sum_{x, \delta}^{m}(-1)^{k} \cdot\left(\begin{array}{c}
m \\
k
\end{array}\right) \cdot U(k \delta),
$$

where $\left(\begin{array}{c}m \\ k\end{array}\right)$ denotes the binomial coefficient $m ! /[k !(m-k) !] .{ }^{3}$ From this we may derive

Theorem. An expected utility maximizer with continuous von Neumann-Morgenstern utility function $U(\cdot)$ will rank probability distributions on the basis of their first $m$ absolute moments if and only if $U(\cdot)$ is a polynomial of at most degree $m$.

Proof. Let $x$ and $\delta$ be arbitrary real numbers, and define the probability distributions ${ }^{4} Y$ and $Z$ by

$$
\begin{gathered}
\operatorname{prob}\{Y=x+k \delta\}=2^{-m} \cdot\left(\begin{array}{c}
m \\
k
\end{array}\right) \text { for } k=0, \ldots, m, k \text { even, } \\
\operatorname{prob}\{Y=k \delta\}=2^{-m} \cdot\left(\begin{array}{c}
m \\
k
\end{array}\right) \text { for } k=0, \ldots, m, k \text { odd }
\end{gathered}
$$

and

$$
\operatorname{prob}\{Z=k \delta\}=2^{-m} \cdot\left(\begin{array}{c}
m \\
k
\end{array}\right) \text { for } k=0, \ldots, m, k \text { even, }
$$

$\operatorname{prob}\{Z=x+k \delta\}=2^{-m} \cdot\left(\begin{array}{c}m \\ k\end{array}\right)$ for $k=0, \ldots, m, k$ odd.

For $l=1, \ldots, m$ the $l$ th absolute moments of $Y$ and $Z$ are given by

$$
\mathrm{E}\left[Y^{l}\right]=\sum_{\substack{k=0 \\
k \text { even }}}^{m}(x+k \delta)^{l} \cdot 2^{-m} \cdot\left(\begin{array}{c}
m \\
k
\end{array}\right)+\sum_{\substack{k=0 \\
k \text { odd }}}^{m}(k \delta)^{l} \cdot 2^{-m} \cdot\left(\begin{array}{c}
m \\
k
\end{array}\right)
$$

and

$$
\mathrm{E}\left[Z^{l}\right]=\sum_{\substack{k=0 \\
k \text { odd }}}^{m}(x+k \delta)^{l} \cdot 2^{-m} \cdot\left(\begin{array}{c}
m \\
k
\end{array}\right)+\sum_{\substack{k=0 \\
k \text { even }}}^{m}(k \delta)^{l} \cdot 2^{-m} \cdot\left(\begin{array}{c}
m \\
k
\end{array}\right),
$$

so that

$$
\begin{aligned}
\mathrm{E}\left[Y^{l}\right]-\mathrm{E}\left[Z^{l}\right] & =\sum_{k=0}^{m}(-1)^{k} \cdot(x+k \delta)^{l} \cdot 2^{-m} \cdot\left(\begin{array}{c}
m \\
k
\end{array}\right)-\sum_{k=0}^{m}(-1)^{k} \cdot(k \delta)^{l} \cdot 2^{-m} \cdot\left(\begin{array}{c}
m \\
k
\end{array}\right) \\
& =\sum_{k=0}^{m}(-1)^{k} \cdot\left[(x+k \delta)^{l}-(k \delta)^{l}\right] \cdot 2^{-m} \cdot\left(\begin{array}{c}
m \\
k
\end{array}\right) .
\end{aligned}
$$

3 Evaluating eq. (6) at $x=w+\delta$ and at $x=w$ and subtracting yields that the $(m+1)$ th difference of $U(\cdot)$ at $w$ will be 0 for all $w$ and $\delta$, which from Aczèl $(1966$, p. 130) implies that $U(\cdot)$ must be a polynomial of at most degree $m$.

4 That the probabilities in (7) and (8) will sum to unity follows from Feller (1968, p. 51, eq. 8.12). 
Substituting

$$
(x+k \delta)^{l}=\sum_{i=0}^{l}\left(\begin{array}{l}
l \\
i
\end{array}\right) \cdot x^{l-i} \cdot(k \delta)^{i}=\sum_{i=0}^{l-1}\left(\begin{array}{l}
l \\
i
\end{array}\right) \cdot x^{l-i} \cdot(k \delta)^{i}+(k \delta)^{l}
$$

yields

$$
\begin{aligned}
\mathrm{E}\left[Y^{l}\right]-\mathrm{E}\left[Z^{l}\right] & =\sum_{k=0}^{m}(-1)^{k} \cdot\left[\sum_{i=0}^{l-1}\left(\begin{array}{l}
l \\
i
\end{array}\right) \cdot x^{l-i} \cdot(k \delta)^{i}\right] \cdot 2^{-m}\left(\begin{array}{c}
m \\
k
\end{array}\right) \\
& =\sum_{i=0}^{l-1}\left(\begin{array}{l}
l \\
i
\end{array}\right) \cdot 2^{-m} \cdot x^{l-i} \cdot \delta^{i} \cdot\left\{\sum_{k=0}^{m}(-1)^{k} \cdot k^{i} \cdot\left(\begin{array}{c}
m \\
k
\end{array}\right)\right\}
\end{aligned}
$$

But since the expression in braces will be 0 for all $i \leq l \cdot 1<m$ [e.g., Feller (1968, p. 65, eq. 12.17)], it follows that the first $m$ moments of $Y$ and $Z$ are equal.

Since the individual evaluates probability distributions on the basis of their first $m$ moments, $Y$ and $Z$ must yield equal expected utility, so that we have:

$$
\begin{aligned}
0=\mathrm{E}[U(Y)]-\mathrm{E}[U(Z)]= & \sum_{\substack{k=0 \\
k \text { even }}}^{m} U(x+k \delta) \cdot 2^{-m} \cdot\left(\begin{array}{c}
m \\
k
\end{array}\right)+\sum_{\substack{k=0 \\
k \text { odd }}}^{m} U(k \delta) \cdot 2^{-m} \cdot\left(\begin{array}{c}
m \\
k
\end{array}\right) \\
& -\sum_{\substack{k=0 \\
k \text { odd }}}^{m} U(x+k \delta) \cdot 2^{-m} \cdot\left(\begin{array}{c}
m \\
k
\end{array}\right)-\sum_{\substack{k=0 \\
k \text { even }}}^{m} U(k \delta) \cdot 2^{-m} \cdot\left(\begin{array}{c}
m \\
k
\end{array}\right),
\end{aligned}
$$

which in turn implies

$$
\sum_{k=0}^{m}(-1)^{k} \cdot\left(\begin{array}{c}
m \\
k
\end{array}\right) \cdot U(x+k \delta) \equiv \sum_{x, \delta} \sum_{k=0}^{m}(-1)^{k} \cdot\left(\begin{array}{c}
m \\
k
\end{array}\right) \cdot U(k \delta) .
$$

But since the left and right sides of (14) are the $m$ th differences of $U(\cdot)$ at $x$ and 0 respectively [or in other words, since (14) is the same as (6)], it follows that $U(\cdot)$ must be a polynomial of at most degree $m$.

\section{References}

Aczèl, J., 1966, Lectures on functional equations and their applications (Academic Press, New York).

Bierwag, G., 1974. The rationale of the mean-standard deviation analysis: Comment. American Economic Review 64, $431-433$.

Borch, K., 1969, A note on uncertainty and indifference curves, Review of Economic Studies 36, 1-4.

Borch, K., 1973, Expected utility expressed in terms of moments, Omega 1, 331-343.

Borch, K, 1974, The rationale of the mean-standard deviation analysis: Comment, American Fconomic Review 64, 428-430

Feller, W., 1968, An introduction to probability theory and its applications, Vol. I (Wiley, New York).

Hanson, D. and C. Menezes, 1971, On a neglected aspect of the theory of risk aversion, Western Economic Journal 9 , $211-217$.

Levy, H., 1974, The rationale of the mean-standard deviation analysis: Comment, American Economic Review 64, $434-441$.

Machina, M. and M. Rothschild, 1987, Risk, in: J. Eatwell, M. Milgate and P. Newman, eds., The new Palgrave: A dictionary of cconomics (The Macmillan Press, London). 
Samuelson, P., 1970, The fundamental approximation theorem of portfolio analysis in terms of means, variances and higher moments, Review of Economic Studies 37, 537-542.

Scott, R. and P. Horvath, 1980, On the direction of preference for moments of higher order than the variance, Journal of Finance 35, 915-919.

Tobin, J., 1958, Liquidity preference as behavior toward risk, Review of Economic Studies 25, 65-86.

Tobin, J., 1969, Comment on Borch and Feldstein, Review of Economic Studies 35, 13-14.

Tsiang, S., 1972, The rationale of the mean-standard deviation analysis, skewness preference, and the demand for money, American Economic Review 62, 354-371.

Tsiang, S., 1974, The rationale of the mean-standard deviation analysis: Reply and errata for the original article, American Economic Review 64, 442-450. 